\title{
Anti-stress effects of combined glucocorticoid and mineralocorticoid receptor blockade in the paraventricular nucleus of the hypothalamus
}

\author{
Hui Ding ${ }^{1}$, Xiang-Yu Cui ${ }^{1}$, Yu-Tong $\mathrm{Liu}^{1}$, Su-Ying $\mathrm{Cui}^{1}$, Xiao Hu ${ }^{1}$, Hui-Ling Zhao ${ }^{1}$, Yu \\ Qin $^{1}$, Nurhumar Kurban ${ }^{1}$, and Yong-He Zhnag ${ }^{1}$ \\ ${ }^{1}$ Peking University Health Science Centre
}

July 22, 2020

\begin{abstract}
Background and Purpose: Mineralocorticoid receptors (MRs), glucocorticoid receptors (GRs) and corticotropin-releasing factor $(\mathrm{CRF})$ in the paraventricular nucleus of the hypothalamus (PVN) are implicated in the stress response. The present study investigated the role of GRs and MRs in the PVN in regulating depressive and anxiety-like behaviors. Experimental Approach: To model chronic stress, rats were exposed to chronic corticosterone treatment via drinking water for 21 days, and the GR antagonist RU486 and MR antagonist spironolactone, alone and combined, were directly injected in the PVN daily for 7 days before the behavioral tests. Depressive- and anxiety-like behaviors were evaluated in forced swim test, sucrose preference test, novelty-suppressed feeding test and social interaction test. The expression of GRs, MRs and CRF were detected by WesternBlot. Key Results: The rats exposed to corticosterone exhibited depressive- and anxiety-like behaviors. The expression of GRs and MRs decreased, and CRF levels increased in the PVN. The intra-PVN administration of RU486 increased the levels of GRs and CRF without influencing depressive- or anxiety-like behaviors. The spironolactone-treated group exhibited an increase in MRs without influencing GRs and CRF in the PVN, and improved anxiety-like behaviors. Interestingly, the intraPVN administration of RU486 and spironolactone combined restored the expression of GRs, MRs, and CRF and improved depressive- and anxiety-like behaviors. Conclusion and Implications: These results suggest that the simultaneous restoration of GRs, MRs, and CRF in the PVN in this rat model of stress might play an important role in the treatment of depression and anxiety.
\end{abstract}

\section{Introduction}

The hypothalamic-pituitary-adrenal (HPA) axis is the driver of neuroendocrine stress responses. In response to stress, neurons in the parvocellular domain of the paraventricular nucleus of the hypothalamus (PVN) are activated to release corticotropin-releasing factor (CRF) and arginine vasopressin (Jiang et al., 2019). CRF coordinates with arginine vasopressin to stimulate anterior pituitary corticotrophs to release adrenocorticotropic hormone (ACTH) into the circulatory system, and ACTH targets the zona fasciculata of the adrenal glands, resulting in the synthesis and secretion of glucocorticoids (GCs; cortisol in humans, corticosterone [CORT] in rodents) (Ulrich-Lai \& Herman, 2009). Glucocorticoids regulate HPA axis activity by limiting their own secretion via a negative feedback mechanism by acting on mineralocorticoid receptors (MRs) and glucocorticoid receptors (GRs) (Binder \& Nemeroff, 2010). These receptors are expressed in neurons that innervate the PVN trans-synaptically and are also found in the PVN (Chen et al., 2014). In the rat PVN, the co-localization of GRs and MRs was found in the parvocellular region, where CRF neurons mainly reside (Han et al., 2005).

The PVN is one of the primary sites of GC negative feedback regulation of the HPA axis. As the central driving force, CRF-producing neurons in the PVN play a key role in determining the state of activation 
of the HPA axis and are closely involved in stress responses and the pathogenesis of depression (Lloyd \& Nemeroff, 2011). Although the exact functional mechanism of GRs and MRs in the modulation of CRF expression remains obscure, MRs and GRs may exert differential regulatory effects on the expression of CRF and operate synergistically in feedback actions of GCs. As the final common pathway for stress integration by the brain, the PVN is ultimately responsible for both normal and pathological features of HPA axis stress responses. Despite progress to date, still unclear are the mechanisms that underlie chronic stress-related PVN drive, the modification of PVN secretagogue signaling, and synaptic plasticity.

Long periods of stress influence the function of GRs and MRs. However, the precise functions of GRs and MRs in the PVN and possible changes that occur in the pathogenesis of depression remain poorly understood (Nguyen et al., 2017), thus hampering our ability to develop strategies to mitigate stress and diseases that are related to stress adaptation.

The predominant hypothesis that CRF hypersecretion plays a leading role in the pathogenesis of major depression has received support from studies that showed that severely depressed patients exhibited high cerebrospinal fluid (CSF) levels of CRF (Gold et al., 2002) and postmortem studies of depressed suicide victims that found very high CSF CRF levels (Pandey et al., 2019). Consistent with the finding that patients with major depression have the highest incidence of HPA axis hyperactivity, studies of depressed suicide victims have revealed high CRF mRNA expression and CRF immunoreactivity in hypothalamic PVN neurons (Deussing \& Chen, 2018).

Glucocorticoid receptors, MRs, and CRF are strongly implicated in the stress response. In the present study, we hypothesized that the regulation of GRs and MRs in the PVN may influence depressive-like behaviors by modulating CRF in the PVN. We investigated the functions of GRs and MRs and their actions on CRF production in the PVN during chronic stress by exploring the behavioral effects of subcutaneous injections or intra-PVN infusions of the GR antagonist RU486 (mifepristone) and MR antagonist spironolactone. We utilized a rat model of depression that was induced by 21 days of CORT administration via drinking water, which produces a series of depressive- and anxiety-like behaviors. We first tested a series of depressive-like behaviors in the forced swim test (FST), sucrose preference test (SPT), novelty-suppressed feeding test (NSFT), and social interaction test (SIT). We then measured the levels of GRs, MRs, and CRF in the PVN. Finally, we co-administered spironolactone and RU486 both subcutaneously and directly in the PVN to evaluate possible correlations between these depressive- and anxiety-like behaviors and changes in GRs, MRs, and CRF in the PVN.

\section{Materials and Methods}

\section{Animals}

Male Wistar rats (Grade I) were purchased from the Animal Center of Peking University (Beijing, China). The animals weighed 250-270 $\mathrm{g}$ at the beginning of the experiments. The rats were maintained on a 12 $\mathrm{h} / 12 \mathrm{~h}$ light/dark cycle (lights on at 09:00) with ad libitum access to food and water. The rats were housed individually in plastic cages. The animal protocols were approved by the Peking University Committee on Animal Care and Use (permission no. LA 2015101).

\section{Surgery for cannula implantation}

Under xylazine $(6 \mathrm{mg} / \mathrm{kg}$, i.p. $)$ and ketamine $(80 \mathrm{mg} / \mathrm{kg}$, i.p. $)$ anesthesia, the rats were implanted with a double-guide cannula (27-gauge; Plastics One, Roanoke, VA, USA) for drug administration into the PVN. Briefly, the cannula was implanted with the tip $0.5 \mathrm{~mm}$ above the PVN (anterior/posterior, $-1.8 \mathrm{~mm}$; medial/lateral, $\pm 0.4 \mathrm{~mm}$; dorsal/ventral, $-7.0 \mathrm{~mm}$ ). The guide cannula was secured with acrylic cement and two stainless steel screws that were anchored to the skull. The cannula placements were histologically verified using Nissl staining of $30 \mu \mathrm{m}$ thick sections by light microscopy (Supplementary Fig. S1).

\section{Drugs administration}


CORT powder (corticosterone, TCI, Tokyo, Japan) was dissolved in $100 \%$ ethanol and then added to the drinking water to a final concentration of $200 \mu \mathrm{g} / \mathrm{mL}$ and $1 \%$ ethanol. Vehicle is $1 \%$ ethanol in the drinking water. Water bottles were wrapped in tinfoil to avoid light-induced degradation of the CORT. Freshly prepared vehicle or CORT was replaced every 2 days. From 21:00 on day 1 to 21:00 on day 22, the rats were exposed to vehicle or CORT for 21 days via drinking water. At an average water intake of $50 \mathrm{~mL} / \mathrm{rat} /$ day, the corresponding daily dose of CORT was $10 \mathrm{mg}$. There was no difference in average daily water intake across the groups. The administration of CORT via drinking water significantly increased circulating CORT levels and induced the absence of circadian rhythm of CORT. This chronic elevation of CORT dysregulates the HPA axis, which is associated clinical depression and anxiety. Preliminary results showed that the activation of HPA axis and the behavioral parameters were not affected by the 21 days of $1 \%$ ethanol exposure.

The GR antagonist RU486 (M1732, Tokyo Chemical Industry, Tokyo, Japan) was dissolved in 1\% ethanol, which $0.9 \%$ saline was added to a final concentration of $1 \mu \mathrm{g} / \mu \mathrm{l}$. The MR antagonist spironolactone (ab141289, Abcam, Shanghai, China) was dissolved in 1\% ethanol, which $0.9 \%$ saline was added to a final concentration of $0.2 \mu \mathrm{g} / \mu \mathrm{l}$. From day 16 to day 22 , drug or vehicle was injected once daily into the PVN in a $0.25 \mu \mathrm{l}$ volume over $2 \mathrm{~min}$ at 08:00 09:00. The injection cannula was kept in place for an additional 2 min to allow the drug to completely diffuse from the tip. The rats received intra-PVN injections of RU486 and spironolactone, alone or combined.

\section{Behavioral tests}

The experimental design is presented in Fig. 1. Behavioral testing was conducted on day 22 and 23. Noveltysuppressed feeding test was performed at 09:00 on day 22. Social interaction test was performed at 20:00 on day 22. Forced swim test was performed at 21:00 on day 22. Sucrose preference test was performed from 22:00 on day 22 to 10:00 on day 23. The rats were decapitated after the behavioral tests on day 23 .

The novelty-suppressed feeding test (NSFT) was performed in an open field arena that contained five food pellets that were placed in the middle of the arena. The food deprivation started at 17:00 on day 21 and lasted for 16 hours. At 09:00 on day 22, individual rats were placed in the corner of the arena and allowed to freely explore it for $5 \mathrm{~min}$. A food pellet was then placed in the center of the arena. The latency for the rat to leave the corner of the box and reach the food was recorded. The increase in latency to feed reflects anxiety-like phenotype. After the NSFT, the rats were returned to their home cages and allowed to eat food. There was no difference in food consumption within 60 min across the groups.

To evaluate sociability, the social interaction test (SIT) was performed as previously described (File \& Seth, 2003). Social interaction test was performed at 20:00 on day 22. Each rat was placed for 5 min in an open field, facing the opposite corner. A novel unfamiliar rat of the same sex and similar weight was also in the arena. The time spent engaged in social interaction (e.g., sniffing, following, and grooming the partner) and total time spent moving around the arena (i.e., exploration) were recorded. The decrease in social interaction reflects anxiety-like phenotype.

Depressive-like behavior was assessed in the forced swim test (FST) as previously described (Porsolt et al,1977; Wang et al., 2015). For adaptation to swimming, on day 21 of CORT exposure, the rats were individually placed for $15 \mathrm{~min}$ in a $25 \mathrm{~cm}$ diameter $\times 60 \mathrm{~cm}$ height Plexiglas cylinder that was filled with $25^{\circ} \mathrm{C} \pm 1{ }^{\circ} \mathrm{C}$ water to a depth of $40 \mathrm{~cm}$. At $21: 00$ on day 22 , each rat was placed in the cylinder for $5 \mathrm{~min}$, and behavior was recorded with video cameras that were oriented in different directions (top and side). Immobility time was assessed from the videotapes by a researcher who was blind to each rat's treatment condition. Immobility was defined as the minimum movement that was necessary to keep the rat's head above water. After the experiment, the rat was removed from the cylinder, dried with a towel, returned to its home cage, and further warmed and dried under a heat lamp. The water in the swim tank was changed for each rat.

Sucrose preference is considered an index of anhedonia (Snyder et al., 2011). Sucrose preference was performed before (baseline) and after chronic CORT exposure. Sucrose preference test (SPT) was conducted in a 5-day sucrose preference protocol. Briefly, rats were individually housed and habituated to two identical bottles 
filled with water on days 1 and 2, then replaced to two identical bottles filled with $1 \%$ sucrose solution on days 3 and 4 . On day 5 , rats were given a free choice between two bottles for $12 \mathrm{~h}$, one filled with $1 \%$ sucrose solution and the other filled with water. The position of the bottles was switched in the middle of the test $(6 \mathrm{~h})$. The sucrose training and baseline test was performed 5 days before the CORT exposure. There was no difference in the sucrose preference across the groups in the baseline test. After 21 days of vehicle or CORT exposure, the SPT was performed for $12 \mathrm{~h}$ beginning at 22:00 on day 22 to 10:00 on day 23. Sucrose preference was calculated according to the following formula: Sucrose preference $(\%)=$ sucrose intake $(\mathrm{g}) /($ sucrose intake $[\mathrm{g}]+$ water intake $[\mathrm{g}]) \times 100 \%$.

\section{Western Blot}

Rats were decapitated after the behavioral tests. Immediately after decapitation, the brains were quickly removed to a pre-chilled brain matrix. Bilateral punches ( $2 \mathrm{~mm}$ diameter) of the PVN (from bregma, $-1.8 \mathrm{~mm}$ to $-1.9 \mathrm{~mm}$ ) and surrounding tissue were made with a hypodermic needle (12 gauge), guided by the Paxinos and Watson rat brain atlas (Paxinos \& Watson, 1998). The process was performed on ice. The samples were stored in pre-chilled microcentrifuge tubes at $-80{ }^{\circ} \mathrm{C}$ until the assay. The PVN tissues were sonicated in $0.5 \mathrm{ml}$ of buffer $/ 100 \mathrm{mg}$ tissue. PVN protein was extracted and boiled in $1 \%$ sodium dodecyl sulfate (SDS) solution and quantified using a BCA assay kit (Pierce, Rockford, IL, USA) with bovine serum albumin as the standard. Equal amounts of protein $(25 \mu \mathrm{g})$ were separated by SDS-polyacrylamide gel electrophoresis on an $8-12 \%$ gradient polyacrylamide gel and transferred to polyvinylidene difluoride membranes (Millipore, Billerica, MA, USA). The membranes were blocked with $5 \%$ skim milk for $1 \mathrm{~h}$ at room temperature and incubated with primary antibodies, including anti- $\beta$-actin (1:1000; Cell Signaling Technology, Danvers, MA, USA), anti-GR (1:500; sc-1004, Santa Cruz Biotechnology, Santa Cruz, CA, USA), anti-MR (1:1000; sc71554, Santa Cruz Biotechnology, Santa Cruz, CA, USA), and anti-CRF (1:800; ab8901, Abcam, Shanghai, China) in TBS-T buffer (Tris-buffered saline $+0.1 \%$ Tween-20) at $4{ }^{\circ} \mathrm{C}$ overnight. After $3 \times 10$ min TBS-T washes, the blots were incubated with horseradish peroxidase-conjugated secondary antibodies (1:2000; Cell Signaling Technology, Danvers, MA, USA) for $2 \mathrm{~h}$ at room temperature. After $3 \times 10 \mathrm{~min}$ TBS-T washes, the blots were developed with a chemiluminescence detection kit (Millipore, Billerica, MA, USA). Western blot bands were scanned with the GelDoc XR System (Bio-Rad, Hercules, CA, USA) and subsequently analyzed densitometrically using Image Lab software. The results were normalized to the protein expression level of $\beta$-actin.

\section{The experimental design and statistical analysis}

Data and statistical analysis comply with the recommendations of the British Journal of Pharmacology on experimental design and analysis in pharmacology (Curtis et al., 2018). In behavioral test, rats were equally assigned to two or four groups with simple randomization in each experiment. In intra-PVN administration experiments, the injection sites were verified by visual inspection or Nissl staining. The data from the animals whose injection site without in the PVN was excluded. The data was analyzed by a person who was blind to the treatments. The data are expressed as the mean \pm SEM and analyzed by GraphPad Prism 8.0 (GraphPad Software Inc., San Diego, CA). The data presented in Fig. 2 was analyzed by unpaired Student's $t$-test. The data presented in Fig. 3-5 was analyzed by one-way analysis of variance (ANOVA) followed by the Tukey's post hoc test. Post hoc tests were conducted only if $F$ in ANOVA achieved $p<0.05$ and there was no significant variance inhomogeneity. The level of statistical significance was set at $p<0.05$.

\section{Results}

Effects of chronic CORT administrationvia drinking water on behavior and GR, MR, and CRF levels in rats

We previously reported that rats that were exposed to CORT via drinking water for 21 days exhibited 
despair, anhedonia, anxiety, and sleep impairments (Ding et al., 2018). However, unknown was whether GRs, MRs, and CRF (i.e., the central component of the stress response) in the PVN are involved in its etiological mechanisms. We reconfirmed that the rats that received CORT via drinking water for 21 days exhibited a significant increase in immobility time in the FST $\left(t_{16}=5.562, p<0.01\right.$, Fig. $\left.2 \mathrm{~A}\right)$ and a significant decrease in sucrose preference in the SPT $\left(t_{16}=8.441, p<0.01\right.$, Fig. $\left.2 \mathrm{~B}\right)$. As shown in Fig. $2 \mathrm{C}$, rats that received CORT exhibited less social interaction in the SIT $\left(t_{16}=7.621, p<0.01\right)$. In the NSFT, chronic CORT consumption significantly prolonged the latency to feed, reflecting anxiety-like behavior $\left(t_{16}\right.$ $=6.305, p<0.01$, Fig. 2D). These data indicate that rats that received chronic CORT via drinking water exhibited both depressive- and anxiety-like behaviors. The Western blot analysis revealed that after chronic CORT treatment, the expression of CRF in the PVN significantly increased $\left(t_{8}=11.98, p<0.01\right.$, Fig. $\left.2 \mathrm{H}\right)$, and both MR $\left(t_{8}=20.07, p<0.01\right.$, Fig. $\left.2 \mathrm{E}\right)$ and GR $\left(t_{8}=9.315, p<0.01\right.$, Fig. $\left.2 \mathrm{~F}\right)$ levels simultaneously decreased in the PVN, but the MR/GR ratio was unchanged (Fig. 2G).

\section{Effects of intra-PVN infusion of RU486 on behavior in rats that were chronically treated with} CORT

We investigated the effects of RU486 on depressive- and anxiety-like behaviors and the levels of GRs, MRs, and CRF in the PVN in rats that were treated with CORT + vehicle. On day 16 during CORT consumption at 08:00, the rats received an intra-PVN infusion of RU486 for 7 days. As shown in Fig. 3, rats that were treated with CORT + vehicle also exhibited depressive-like behaviors in the FST $\left(F_{3,23}=42.47, p<0.01\right.$, Fig. 3A) and SPT $\left(F_{3,23}=7.599, p<0.01\right.$, Fig. 3B $)$ and anxiety-like behaviors in the $\operatorname{SIT}\left(F_{3,23}=24.03\right.$, $p<0.01$, Fig. 3C) and NSFT $\left(F_{3,23}=6.225, p<0.01\right.$, Fig. 3D). The intra-PVN infusion of RU486 did not influence CORT-induced depressive- or anxiety-like behaviors (Fig. 3A-D). Western blot revealed that the intra-PVN infusion of RU486 restored GR expression in the PVN $\left(F_{3,16}=15.88, p<0.01\right.$, Fig. 3F) without marked improvement in MR levels in the PVN (Fig. 3E) and significantly decreased the MR/GR ratio $\left(F_{3,16}=8.628, p<0.01\right.$, Fig. $\left.3 \mathrm{G}\right)$. Unexpectedly, intra-PVN RU486 administration augmented the increase in CRF levels compared with rats that were treated with CORT + vehicle $\left(F_{3,16}=94.75, p<\right.$ 0.01, Fig. $3 \mathrm{H})$. These data suggest that although GR levels in the PVN in rats that were treated with CORT + vehicle rats were restored to normal by the intra-PVN infusion of RU486 alone, the MR/GR ratio was still imbalanced, and CRF levels remained high. Thus, depressive- and anxiety-like behaviors in rats that were chronically treated with CORT were not inhibited.

Effects of intra-PVN infusion of spironolactone on behavior in rats that were chronically treated with CORT

To elucidate the involvement of MRs in the PVN in the behavioral alterations that were induced by chronic CORT exposure, the MR antagonist spironolactone was microinjected into the PVN on day 16 of CORT consumption at 08:00 for 7 days. Depressive-like behavior, including immobility time in the FST (Fig. 4A) and sucrose preference in the SPT (Fig. 4B), were not reversed by intra-PVN spironolactone administration. In contrast, the intra-PVN infusion of spironolactone in CORT-exposed rats exerted anxiolytic-like effects, reflected by an increase in social interaction in the SIT $\left(F_{3,22}=18.76, p<0.01\right.$, Fig. $\left.4 \mathrm{C}\right)$ and a shorter feeding latency in the NSFT $\left(F_{3,22}=19.07, p<0.01\right.$, Fig. 4D). The intra-PVN injection of spironolactone reversed the decrease in MR levels in the PVN that was induced by chronic CORT exposure $\left(F_{3,16}=26.15\right.$, $p<0.01$, Fig. 4E). Chronic CORT exposure significantly altered GR and CRF expression, and these changes were unaffected by intra-PVN spironolactone administration (Fig. 4F, H). Because MR levels returned to normal but GRs levels failed to recover, the MR/GR ratio was two-times higher than normal and notably out of balance $\left(F_{3,16}=7.344, p<0.01\right.$, Fig. $\left.4 \mathrm{G}\right)$. Based on these results, the CORT-induced decrease in MR levels in the PVN may be associated with anxiety-like behavior, and intra-PVN spironolactone administration restored MR levels to normal, which may be related to its anxiolytic effect. Meanwhile, the failure of GR and CRF levels and the MR/GR ratio to return to normal in the PVN in CORT-treated rats may explain why the intra-PVN infusion of spironolactone did not produce an antidepressant effect.

Effects of co-administration of RU486 and spironolactone in the PVN on behavior and GR, MR and CRF levels in rats that were chronically treated with CORT 
Accumulating evidence indicates that MRs cooperate with GRs in the stress response (Spencer et al., 1998; Mattsson et al., 2009; Jadavji et al., 2011). In this experiment, we co-administered the GR antagonist RU486 and MR antagonist spironolactone in the PVN. RU486 was microinjected in the PVN once daily beginning on day 16 of CORT consumption at 08:00 for 7 days. Spironolactone was administered on the same days, 40 min after RU486 administration. Combined GR and MR blockade in the PVN in rats that were chronically treated with CORT restored behavior in the FST $\left(F_{3,22}=15.78, p<0.01\right.$, Fig. 5A $)$, SPT $\left(F_{3,22}=9.56\right.$, $p<0.01$, Fig. 5B), SIT $\left(F_{3,22}=16.68, p<0.01\right.$, Fig. 5C) and NSFT $\left(F_{3,22}=18.99, p<0.01\right.$; Fig. 5D $)$ and the levels of MRs $\left(F_{3,16}=14.64, p<0.01\right.$, Fig. 5E $)$, GRs $\left(F_{3,16}=33.93, p<0.01\right.$, Fig. 5F $)$ and CRF $\left(F_{3,16}=22.60, p<0.01\right.$, Fig. $\left.5 \mathrm{H}\right)$ in the PVN. Altogether, these data suggested co-administration of the MR and GR antagonists in the PVN restored the levels of MRs, GRs, and CRF and the MR/GR ratio in the PVN to normal in CORT-exposed rats, thus inhibiting depressive- and anxiety-like behaviors.

\section{Discussion}

In the present study, we hypothesized that the regulation of GRs and MRs in the PVN may influence depressive-like behaviors by modulating CRF in the PVN. After exogenous CORT exposure via drinking water for 21 days, plasma corticosterone rhythms were clearly altered in the present rat model (Supplementary Fig. S2), which resulted in the overproduction of CRF and reductions of GR and MR expression in the PVN. Intra-PVN administration of only the combination of the GR antagonist RU486 and MR antagonist spironolactone inhibited depressive- or anxiety-like behaviors and restored MR, GR, and CRF levels and the $\mathrm{MR} / \mathrm{GR}$ ratio in the PVN to normal. However, intra-PVN administration of either RU486 or spironolactone alone did not inhibit depressive-like behaviors. Intra-PVN administration of spironolactone alone inhibit anxiety-like behaviors.

Glucocorticoid receptors are richly expressed in the medial parvocellular PVN and co-localize with CRF, thus placing the PVN in a prime position to control the output of neurons that activate the HPA axis (Liposits et al., 1987; Uht et al., 1988). Although the PVN is well known to be the major site of CRF synthesis in the brain (De Souza, 1995), with projections to many extrahypothalamic areas that are involved in various behaviors, its role in stress-related behaviors has not been studied extensively. Chronic variable stress reduces the expression of GRs in the medial parvocellular PVN. CRF expression is negatively regulated by GCs, and GRs may play a role in the observed upregulation of CRF during chronic stress (Herman et al., 1995). This negative correlation between GR and CRF levels (i.e., downregulation of GRs and upregulation of CRF) was also observed in the PVN in the present rat model. However, the intra-PVN infusion of RU486 returned GRs level in the PVN to normal, had no effect on MR, decreased the MR/GR ratio, and increased CRF levels, and depressive- and anxiety-like behaviors did not improve. These results showed that simply increasing the expression of GRs in the PVN without reversing CRF to normal levels could not improve either depressive- or anxiety-like behavior. Therefore, one unresolved issue is why the recovery of GRs did not return CRF levels to normal but rather further increased the abnormally high levels of CRF. One possibility is the recruitment of other mechanisms beyond the negative regulation of CRF by GRs. Lamberts et al. reported that RU486 activated the HPA axis, resulting in a resetting of this system at a higher level at which the diurnal rhythm and responsiveness to CRF stimulation were maintained, whereas the sensitivity to dexamethasone diminished, and these changes were caused by the induction of partial cortisol receptor resistance during RU486 therapy (Lamberts et al., 1991). Based on its mechanism of action and evidence that the restoration of normal hypothalamic CRF activity by RU486 appears to be the rate-limiting step in HPA axis recovery (Muglia et al., 2000), central GR blockade may deprive the hypothalamus from GR activity, thereby stimulating CRF-producing neurons in the hypothalamus and resulting in the further enhancement of CRF.

The HPA axis has long been recognized for its involvement in depression, with a focus on cortisol/CORT and less of an emphasis on aldosterone as a stress hormone. A recent study found that aldosterone acted selectively in mood-regulating brain areas, without competing with cortisol/CORT (Murck et al., 2014). Although receptors for aldosterone (i.e., MRs) are known to be expressed in the PVN, its role in the local regulation of HPA axis function has been less explored. The MR antagonist spironolactone inhibits the 
effects of mineralocorticoids by displacing them from MRs in the PVN. Wu et al. (2013) found that chronic subcutaneous CORT treatment triggered several depressive-like behaviors and downregulated MR expression in parallel in the hippocampus and hypothalamus. On the other hand, they also reported that intraperitoneal spironolactone administration for 7 days in animals that were subcutaneously treated with CORT reduced immobility time in the FST and improved performance in a novel object recognition test (Wu et al., 2013). In the present study, CORT-exposed rats via drinking water also exhibited lower MR expression in the prefrontal cortex, hippocampus, and amygdala (data not shown). Bitran et al. (1998) hypothesized that the anxiolytic effects of an MR antagonist may be more pronounced in animals whose hippocampal MRs are activated by CORT or the MR agonist aldosterone. In the present study, the intra-PVN infusion of spironolactone restored MR levels in the PVN to normal and improved anxiety-like behaviors in the NSFT and SIT, without altering CRF levels in the PVN, but it did not improve depressive-like behaviors in the FST or SPT. However, still needing to be confirmed is whether the anxiolytic effects were caused by the restoration of MRs in the PVN in the present rat model. Thus, further studies should seek to determine the pharmacological specificity of the effects of intra-PVN spironolactone administration to exclude possible actions of spironolactone at sites other than MRs.

The balance between MRs and GRs during chronic stress plays an important role in the etiology of depression (De Kloet et al., 1998). The pharmacological modulation of GRs and MRs also influences the endocrine stress response and depressive-like behaviors in rodents. The ways in which MRs and GRs interact to influence the endocrine system and related behaviors are still being clarified. In the present study, RU486 alone restored the downregulation of GR levels in the PVN to normal but did not influence the downregulation of MRs, thus making the MR/GR ratio significantly lower than normal and failing to suppress depressive- and anxietylike behaviors (Fig. 3). Spironolactone treatment alone restored MR levels in the PVN to normal but did not influence the downregulation of GRs, thus making the MR/GR ratio significantly higher than normal and inhibiting anxiety-like behaviors in the NSFT and SIT (Fig. 4). When RU486 and spironolactone were administered together, MR and GR levels and the MR/GR ratio returned to normal, and depressive- and anxiety-like behaviors significantly improved (Fig. 5). The present findings suggest that the normalization of MR, GR, and CRF levels and the MR/GR ratio may be necessary conditions to inhibit depressive- and anxiety-like behaviors in the present rat model. Nonetheless, the restoration of MR levels in the PVN to normal may be a sufficient condition to improve anxiety-like behaviors.

CRF also acts within other regions of the brain where it directly contributes to stress-responsive behavior. Indeed, some depressed patients had high CSF levels of CRF, and these levels are normalized in patients who respond to antidepressant treatment, suggesting that these alterations of CRF levels contribute to the symptomology of depression (Pandey et al., 2019). In the central nervous system, patients had high levels of CRF in the PVN and in monoaminergic nuclei, including the locus coeruleus and raphe (Austin et al., 2003; Bissette et al., 2003). Elevations of plasma CRF levels in depressed individuals are ameliorated by successful electroconvulsive shock therapy (Nemeroff et al., 1991) and antidepressant treatment (Heuser et al., 1998). The causal relationship between depression and CRF hyperactivity and receptor expression is unclear. The present results suggest that a state of equilibrium between GRs and MRs in the PVN may improve the function of the HPA axis, which can improve depressive- and anxiety-like behaviors. However, the specific ways in which GRs, MRs, and CRF in the PVN are involved in the pathogenesis of depression and anxiety need to be elucidated.

The present study has some limitations. We only examined male rats to eliminate gender influences that are known to modulate experimentally induced emotion and HPA activity. The significance of sex differences in PVN function is virtually unexplored. Therefore, we do not know whether our results are generalizable to female rats. Furthermore, we did not detect the activity of HPA function. Therefore, unclear was the state of the HPA axis when antidepressant effects were induced by GR and MR blockade. One possibility is that the target sites of the antidepressant effects of RU486 and spironolactone may not be in the PVN.

Overall, the present study found that the combination of pharmacological GR and MR antagonism in the PVN prevented the pathogenesis of depressive- and anxiety-like behaviors in rats that were chronically treated 
with CORT, confirming that both MRs and GRs in the PVN play an important role in CRF function. Such a combined pharmacological approach may be a novel strategy to prevent hypercortisolism- or chronic stressinduced depression and its metabolic complications. Much work still needs to be done to achieve a more complete understanding of the role of GRs, MRs, and CRF in the PVN in depressive- and anxiety-like behaviors.

\section{Acknowledgements}

This work was supported by grants from the National Natural Science Foundation of China (No. 81872851 and 81871038) and National Scientific \& Technological major special project (No. 2019ZX09301-127). The authors declare no competing interests.

\section{Author contributions}

H.D. and X.Y.C. designed, conducted, and analyzed the experiments with oversight by Y.H.Z.. Y.T.L. and X.H. performed forced swim test. H.L.Z and Y.Q. performed sucrose preference test. N.K. performed noveltysuppressed feeding test. H.D., S.Y.C. and Y.H.Z. wrote the paper. All authors reviewed and approved the final manuscript.

\section{Conflict of interest statement}

The authors declare no conflicts of interest.

\section{REFERENCES}

Austin, M.C., Janosky, J.E., \& Murphy, H.A. (2003). Increased corticotropin-releasing hormone immunoreactivity in monoamine-containing pontine nuclei of depressed suicide men. Molecular psychiatry ,8 , 324-332.

Binder, E.B., \& Nemeroff, C.B. (2010). The CRF system, stress, depression and anxiety-insights from human genetic studies. Molecular Psychiatry , 15, 574-588.

Bissette, G., Klimek, V., Pan, J., Stockmeier, C., \& Ordway, G. (2003). Elevated concentrations of CRF in the locus coeruleus of depressed subjects. Neuropsychopharmacology , 28 , 1328-1335.

Bitran, D., Shiekh, M., Dowd, J.A., Dugan, M.M., \& Renda, P. (1998). Corticosterone is permissive to the anxiolytic effect that results from the blockade of hippocampal mineralocorticoid receptors.Pharmacology Biochemistry and Behavior , 60 , 879-887.

Chen, J, Gomez-Sanchez, C.E., Penman, A., May, P.J., \& Gomez-Sanchez, E. (2014). Expression of mineralocorticoid and glucocorticoid receptors in preautonomic neurons of the rat paraventricular nucleus. American Journal of Physiology-Regulatory Integrative and Comparative Physiology , 306 , R328-R340.

De Kloet, E.R., Vreugdenhil, E., Oitzl, M.S., \& Joels, M. (1998). Brain Corticosteroid Receptor Balance in Health and Disease. Endocrine Reviews , 19 , 269-301.

De Souza, E.B. (1995). Corticotropin-releasing factor receptors: physiology, pharmacology, biochemistry and role in central nervous system and immune disorders. Psychoneuroendocrinology , 20 , 789-819.

Deussing, J.M., \& Chen, A. (2018). The Corticotropin-Releasing Factor Family: Physiology of the Stress Response. Physiological Reviews ,98 , 2225-2286.

Ding, H., Cui, X.Y., Cui, S.Y., Ye, H., Hu, X., Zhao, H.L., . . Zhang, Y.H. (2018). Depression-like behaviors induced by chronic corticosterone exposure via drinking water: Time-course analysis.Neuroscience Letters , $687,202-206$.

File, S.E., \& Seth, P. (2003). A review of 25 years of the social interaction test. European Journal of Pharmacology , 463, 35-53.

Gold, P.W., Gabry, K.E., Yasuda, M.R., \& Chrousos, G.P. (2002). Divergent endocrine abnormalities in melancholic and atypical depression: clinical and pathophysiologic implications. Endocrinology and 
Metabolism Clinics of North America , 31, 37-62.

Han, F., Ozawa, H., Matsuda, K., Nishi, M., \& Kawata, M., (2005). Colocalization of mineralocorticoid receptor and glucocorticoid receptor in the hippocampus and hypothalamus. Neuroscience Research ,51, $371-381$.

Herman JP, Adams D, \& Prewitt C. (1995). Regulatory changes in neuroendocrine stress-integrative circuitry produced by a variable stress paradigm. Neuroendocrinology , 61 , 180-190.

Heuser, I., Bissette, G., Dettling, M., Schweiger, U., Gotthardt, U., Schmider, J., ... Holsboer, F. (1998). Cerebrospinal fluid concentrations of corticotropin-releasing hormone, vasopressin, and somatostatin in depressed patients and healthy controls: response to amitriptyline treatment. Depression and Anxiety , 8 , $71-79$.

Jadavji, N.M., Supina, R.D., \& Metz, G.A. (2011). Blockade of Mineralocorticoid and Glucocorticoid Receptors Reverses Stress-Induced Motor Impairments. Neuroendocrinology , 94 , 278-290.

Jiang, Z., Rajamanickam, S., \& Justice, N.J. (2019). CRF signaling between neurons in the paraventricular nucleus of the hypothalamus (PVN) coordinates stress responses. Neurobiology of Stress , 11, 100192.

Lamberts, S.W.J., Koper, J.W., \& de Jong, F.H. (1991). The Endocrine Effects of Long-Term Treatment with Mifepristone (RU 486). Journal of Clinical Endocrinology E Metabolism , 73 , 187-191.

Liposits, Z., Uht, R.M., Harrison, R.W., Gibbs, F.P., Paull, W.K., \& Bohn, M.C. (1987). Ultrastructural localization of glucocorticoid receptor (GR) in hypothalamic paraventricular neurons synthesizing corticotropin releasing factor (CRF). Histochemistry , 87, 407-412.

Lloyd, R.B., \& Nemeroff, C.B. (2011). The role of corticotropin-releasing hormone in the pathophysiology of depression: therapeutic implications, Current Topics in Medicinal Chemistry ,11 , 609-617.

Mattsson, C., Reynolds, R.M., Simonyte, K., Olsson, T., \& Walker, B.R. (2009). Combined receptor antagonist stimulation of the hypothalamic-pituitary-adrenal axis test identifies impaired negative feedback sensitivity to cortisol in obese men. Journal of Clinical Endocrinology 83 Metabolism , 94 , 1347-1352.

Muglia, L.J., Bethin, K.E., Jacobson, L., Vogt, S.K., \& Majzoub, J.A. (2000). Pituitary-adrenal axis regulation in CRH-deficient mice.Endocrine Research , 26 , 1057-1066.

Murck, H., Büttner, M., Kircher, T., \& Konrad, C. (2014). Genetic, molecular and clinical determinants for the involvement of aldosterone and its receptors in major depression. Nephron Physiology ,128, 17-25.

Nemeroff, C.B., Bissette, G., Akil, H., \& Fink, M. (1991). Neuropeptide concentrations in the cerebrospinal fluid of depressed patients treated with electroconvulsive therapy. Corticotrophin-releasing factor, betaendorphin and somatostatin. British Journal of Psychiatry ,158, 59-63.

Nguyen, E.T., Streicher, J., Berman, S., Caldwell, J.L., Ghisays, V., Estrada CM, .. Solomon MB. (2017). A mixed glucocorticoid/mineralocorticoid receptor modulator dampens endocrine and hippocampal stress responsivity in male rats. Physiology $\&$ Behavior , 178, 82-92.

Pandey, G.N., Rizavi, H.S., Bhaumik, R., \& Ren, X. (2019). Increased protein and mRNA expression of corticotropin-releasing factor (CRF), decreased CRF receptors and CRF binding protein in specific postmortem brain areas of teenage suicide subjects.Psychoneuroendocrinology , 106 , 233-243.

Paxinos, G.A., Watson, C. (1998). The Rat Brain in Stereotaxic Coordinates. San Diego, California: Academic Press.

Porsolt, R.D., Le Pichon, M., \& Jalfre, M. (1977). Depression: a new animal model sensitive to antidepressant treatments. Nature ,266, 730-732.

Snyder, J.S., Soumier, A., Brewer, M., Pickel, J., \& Cameron, H.A. (2011). Adult hippocampal neurogenesis buffers stress responses and depressive behavior. Nature , 476, 458-461. 
Spencer, R., Kim, P., Klaman, B., \& Cole, M. (1998). Evidence for mineralocorticoid receptor facilitation of glucocorticoid receptor-dependent regulation of hypothalamic-pituitary-adrenal axis activity. Endocrinology , $139,2718-2726$.

Uht, R.M., McKelvy, J.F., Harrison, R.W., \& Bohn, M.C. (1988). Demonstration of glucocorticoid receptorlike immunoreactivity in glucocorticoid-sensitive vasopressin and corticotropin-releasing factor neurons in the hypothalamic paraventricular nucleus. Journal of Neuroscience Research , 19 , 405-469.

Ulrich-Lai, Y.M., \& Herman, J.P. (2009). Neural regulation of endocrine and autonomic stress responses. Nature Reviews Neuroscience ,10 , 397-409.

Wang, Z.J., Zhang, X.Q., Cui, X.Y., Cui, S.Y., Yu, B., Sheng, Z.F., .. Zhang, Y.H. (2015). Glucocorticoid receptors in the locus coeruleus mediate sleep disorders caused by repeated corticosterone treatment. Scientific Reports , 5 , 9442.

Wu, T.C., Chen, H.T., Chang, H.Y., Yang, C.Y., Hsiao, M.C., Cheng, M.L., \& Chen, J.C. (2013). Mineralocorticoid receptor antagonist spironolactone prevents chronic corticosterone induced depression-like behavior. Psychoneuroendocrinology , $38,871-883$.

\section{Figure Legends}

Fig. 1. Experimental design. Rats received surgery of implantation of cannula into PVN. The rats were exposed to the vehicle or CORT via drinking water for 21 days from day 1 to day 22 . The rats received intra-PVN injections of RU486 and spironolactone, alone or combined from day 16 to day 22. The rats underwent behavioral experiments including novelty-suppressed feeding test, social interaction test, forced swim test and sucrose preference test on day 22 and day 23. The rats were decapitated after the behavioral tests on day 23.

Fig. 2. Chronic CORT treatment for 21 days induced depressive- and anxiety-like behaviors, decreased GR and MR protein levels, and increased CRF protein levels in the PVN. Immobility time in the FST (A) and sucrose preference in the SPT (B) were detected to evaluate depressive-like behaviors. Social interaction in the SIT (C) and latency to feed in the NSFT (D) were detected to evaluate anxiety-like behaviors $\left(n_{1}\right.$ , $n_{2}=9$ ). (E-I) Quantification of MRs (E), GRs (F), the MR/GR ratio $(\mathrm{G})$, and CRF $(\mathrm{H})$ in the PVN and representative Western blots (I) after 21 days of vehicle or CORT administration. $\beta$-actin is shown as a quantitative loading control $\left(n_{1}, n_{2}=5\right)$. The data are expressed as mean \pm SEM. ${ }^{*} p<0.05,{ }^{* *} p<0.01$, $v s$. vehicle group (unpaired Student'st -test).

Fig. 3. Intra-PVN administration of RU486 for 7 days before the test had no effect on depressive- or anxietylike behaviors in rats that were chronically exposed to CORT via drinking water for 21 days. Immobility time in the FST (A) and sucrose preference in the SPT (B) were detected to evaluate depressive-like behaviors. Social interaction in the SIT (C) and latency to feed in the NSFT (D) were detected to evaluate anxiety-like behaviors $\left(n_{1}, n_{2}, n_{3}=6, n_{4}=9\right)$. (E-I) Quantification of MRs (E), GRs (F), the MR/GR ratio (G), and $\mathrm{CRF}(\mathrm{H})$ in the PVN and representative Western blots (I). $\beta$-actin is shown as a quantitative loading control $\left(n_{1}, n_{2}, n_{3}, n_{4}=5\right)$. The data are expressed as mean \pm SEM. ${ }^{*} p<0.05,{ }^{*} p<0.01$, vs . vehicle group; ${ }^{\#} p<0.05,{ }^{\# \#} p<0.01, v s$. CORT alone group (one-way ANOVA followed by the Tukey's post hoc test).

Fig. 4. Intra-PVN infusion of spironolactone (SPL) for 7 days before the test ameliorated chronic CORTinduced anxiety-like behaviors. Immobility time in the FST (A) and sucrose preference in the SPT (B) were detected to evaluate depressive-like behaviors. Social interaction in the SIT (C) and latency to feed in the NSFT (D) were detected to evaluate anxiety-like behaviors $\left(n_{1}, n_{2}, n_{3}=6, n_{4}=8\right)$. (E-I) Quantification of GRs (E), MRs (F), the MR/GR ratio (G), and CRF (H) in the PVN and representative Western blots (I). $\beta$-actin is shown as a quantitative loading control $\left(n_{1}, n_{2}, n_{3}, n_{4}=5\right)$. The data are expressed as mean \pm SEM. ${ }^{*} p<0.05,{ }^{* *} p<0.01$, vs . vehicle group; ${ }^{\#} p<0.05,{ }^{\# \#} p<0.01, v s$. CORT alone group (one-way ANOVA followed by the Tukey's post hoc test). 
Fig. 5.Co-administration of RU486 and spironolactone in the PVN produced anxiolytic- and antidepressantlike effects. Immobility time in the FST (A) and sucrose preference in the SPT (B) were detected to evaluate depressive-like behaviors. Social interaction in the SIT (C) and latency to feed in the NSFT (D) were detected to evaluate anxiety-like behaviors $\left(n_{1}, n_{2}, n_{3}=6, n_{4}=8\right)$. (E-I) Quantification of MRs (E), GRs (F), the $\mathrm{MR} / \mathrm{GR}$ ratio $(\mathrm{G})$, and $\mathrm{CRF}(\mathrm{H})$ in the PVN and representative Western blots (I). $\beta$-actin is shown as a quantitative loading control $\left(n_{1}, n_{2}, n_{3}, n_{4}=5\right)$. The data are expressed as mean \pm SEM. ${ }^{*} p<0.05,{ }^{* *} p$ $<0.01$, vs . vehicle group; ${ }^{\#} p<0.05,{ }^{\# \#} p<0.01$, vs . CORT alone group (one-way ANOVA followed by the Tukey's post hoc test).

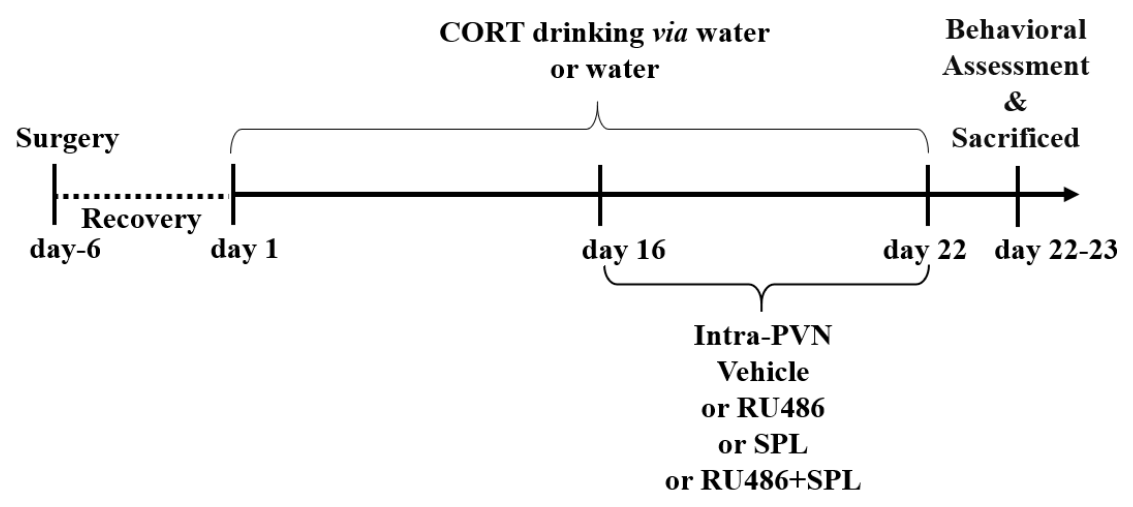



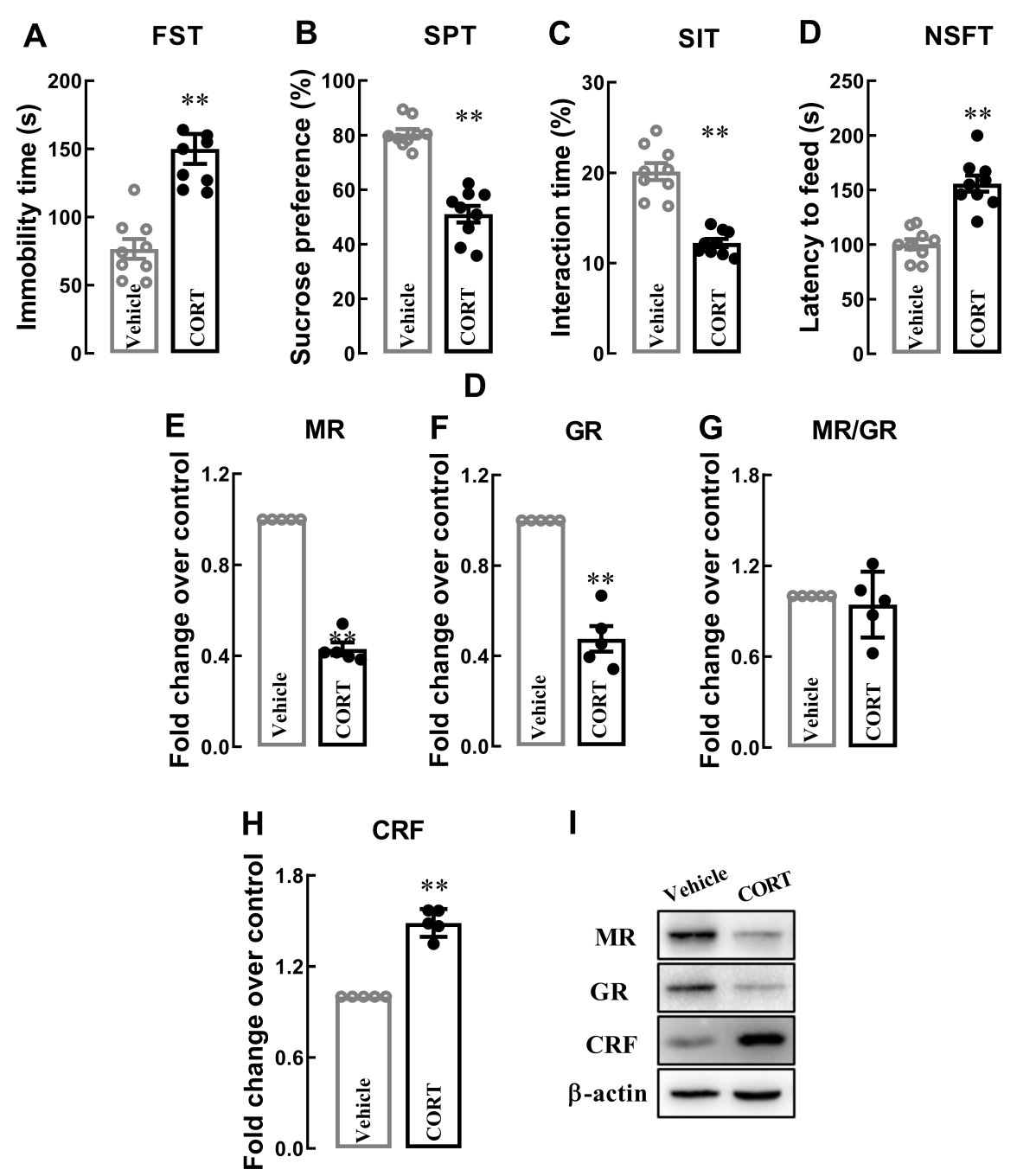

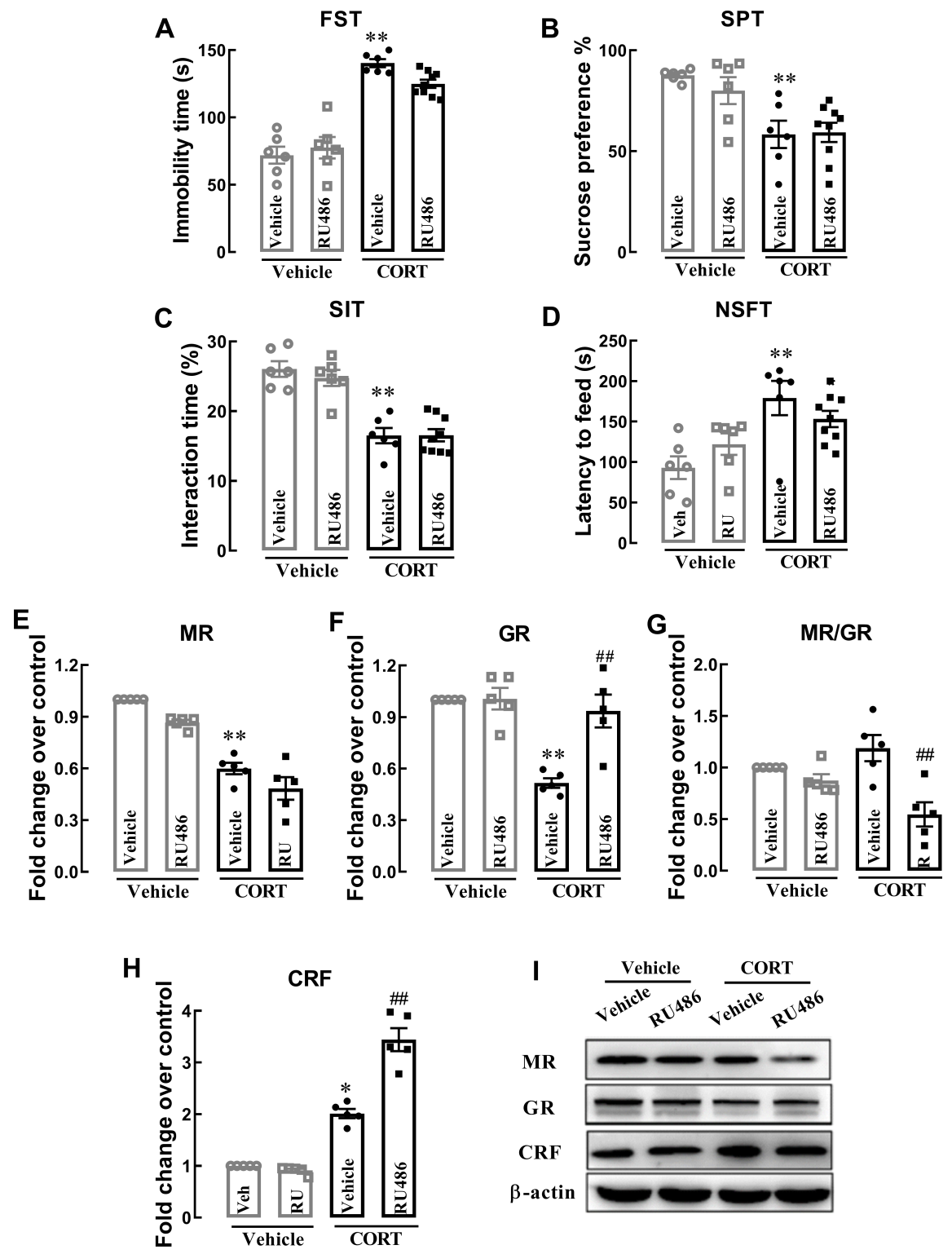


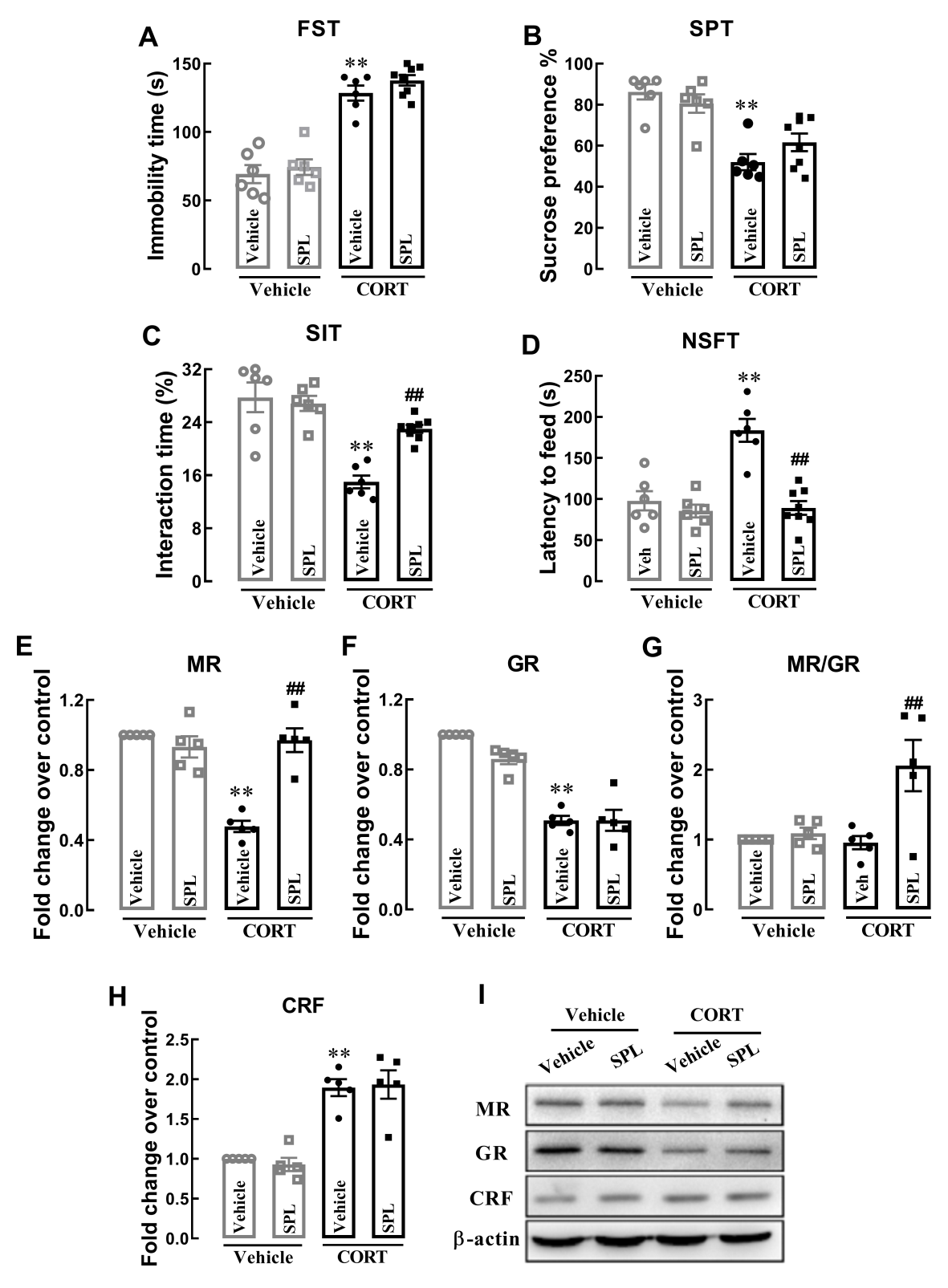



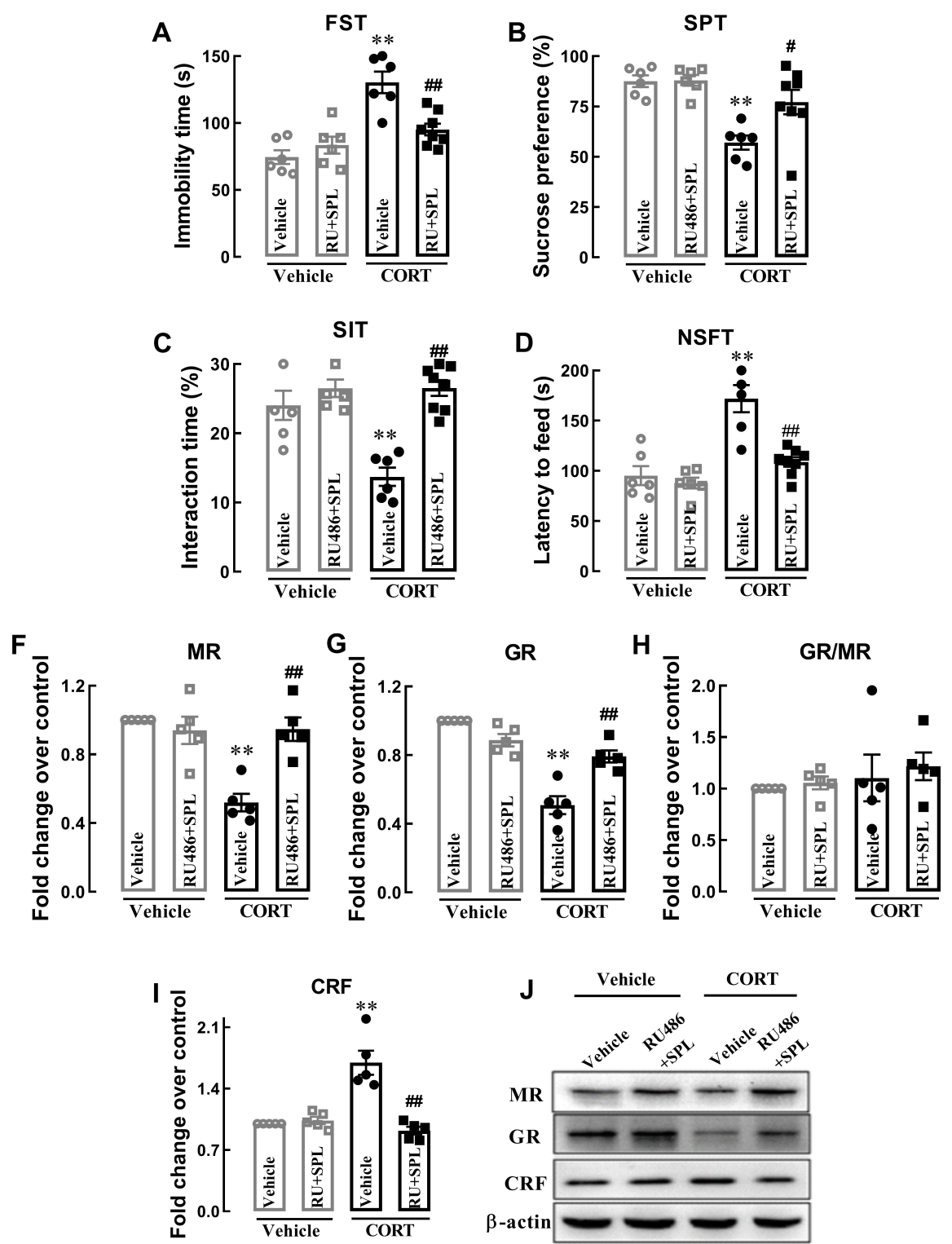